\section{Prostatakarzinom: Lohnt sich Früherkennung auch für Männer über 75?}

Bislang ging man davon aus, dass der Prostatakrebs gerade bei älteren Männern eher langsam wächst und die Patienten schließlich meist aus anderen Gründen versterben. Doch eine amerikanische Studie zeigte jetzt: Von allen Patienten, die wegen ihres Prostatakarzinoms starben, war über die Hälfte älter als 75 Jahre, obwohl in dieser Altersgruppe nur gut ein Viertel aller Tumoren diagnostiziert wurde.

Z iel der vorliegenden Studie war es, zu untersuchen, wie häufig in den verschiedenen Altersgruppen zum Zeitpunkt der Diagnose Prostatakarzinom bereits Metastasen vorlagen und ob ein Zusammenhang zwischen dem Alter der Patienten und der prostatakrebsspezifischen Mortalität bestand. Hierzu wurden die Daten von über 460.000 Amerikanern ausgewertet, bei denen zwischen 1998 und 2007 ein Prostatakarzinom festgestellt worden war.

Die Zahl aller neu diagnostizierten Prostatakarzinome stieg mit dem Alter an und erreichte bei den 70-Jährigen ein Maximum. Danach fiel die Häufigkeit wieder ab. Dies galt allerdings nicht, wenn man die Fälle betrachtete, bei denen sich bereits Metastasen gebildet hatten (M1). Je älter die Patienten bei Diagnosestellung waren, desto häufiger lag ein GleasonScore zwischen 8 und 10 vor und desto öfter war der Tumor bereits metastasiert. Während in der Gruppe der unter 75-Jährigen zum Zeitpunkt der Diagnosestellung nur bei $3 \%$ Metastasen diagnostiziert wurden (M1), waren es bei den 75- bis 79-Jährigen bereits $5 \%$, zwischen 80 und
84 Jahren $8 \%$, in der Gruppe der 85 - bis 89 -Jährigen $13 \%$, und bei den Senioren ab 90 Jahren wurden im Rahmen der Erstdiagnose bei $17 \%$ Metastasen festgestellt.

Entsprechend stieg mit dem Alter die kumulative Inzidenz der Todesfälle infolge des Prostatakarzinoms an, auch wenn die Patienten natürlich auch zunehmend aus anderen Gründen starben. Während die prostatakrebsspezifische Sterberate unter den Patienten, die jünger als 75 Jahre waren, bei 3-4\% lag, verstarben innerhalb von fünf Jahren $7 \%$ der Patienten zwischen 75 und 79 an ihrem Krebs (21\% an anderen Ursachen, aU), $13 \%$ aus der Gruppe der 80 - bis 84 -Jährigen ( $32 \%$ aU), $20 \%$ der 85 - bis 89 -Jährigen ( $43 \%$ aU) und $30 \%$ der Patienten über 90 Jahre (55\% aU). Obwohl nur $26 \%$ der Patienten, bei denen ein Prostatakarzinom diagnostiziert wurde, 75 Jahre oder älter waren, stellten sie fast die Hälfte der Gruppe $(48 \%)$, in der ein fortgeschrittenes Stadium (M1) festgestellt wurde. Und auch in der Gruppe der Krebspatienten, die an ihrem Prostatakarzinom starben, waren die Senioren ab 75 mit einem Anteil von $53 \%$ überrepräsentiert.
Fazit: Ein Grund für die altersabhängigen Unterschiede bei der Erstdiagnose und das höhere Mortalitätsrisiko der Älteren könnte darin liegen, dass bei älteren Männern der Prostatakrebs möglicherweise aggressiver wächst als bisher angenommen, so die Autoren. Allerdings könnte auch eine Rolle spielen, dass Männer über 75 seltener an Früherkennungsuntersuchungen teilnehmen und bei erhöhten PSA-Werten nicht so häufig biopsiert werden wie Jüngere. Vor allem vor dem Hintergrund der Diskussion zum Prostatakarzinom-Screening sollten die Ergebnisse dieser Studie Beachtung finden, denn sie könnten auf den präventiven Nutzen einer frühen Diagnostik auch bei älteren Männern hinweisen. Dr. Christine Starostzik

Scosyrev E et al. Prostate Cancer in the Elderly. Cancer 2012; 118: 3062-70

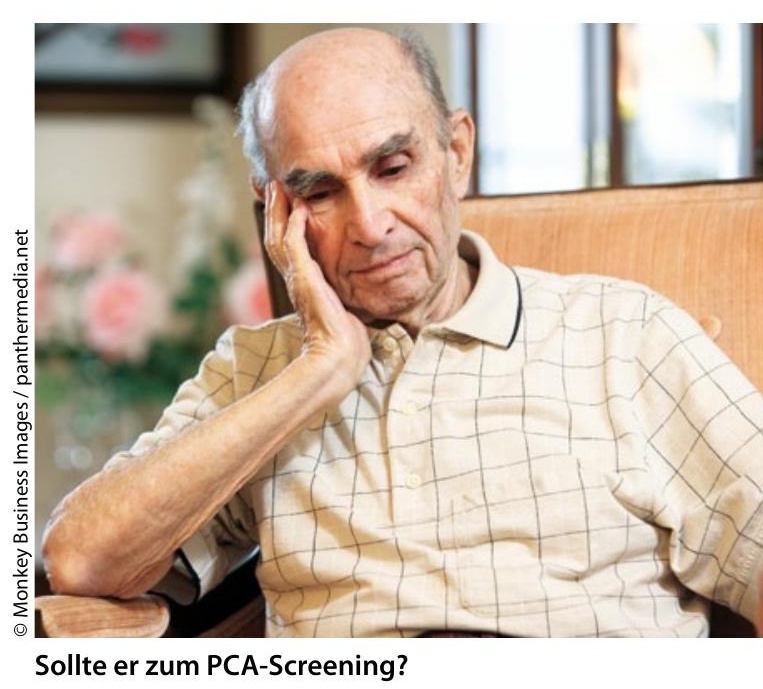

